\title{
Exact solutions for some of the fractional integro-differential equations with the nonlocal boundary conditions by using the modification of He's variational iteration method
}

\author{
S. Irandoust-pakchin a , S. Abdi-Mazraeh b \\ a Faculty of Mathematical Sciences, University of Tabriz, Tabriz, Iran \\ ${ }^{b}$ Department of Mathematics, Azarbaijan Shahid Madani University, Tabriz, Iran
}

\begin{abstract}
In this paper, the modification of He's variational iteration method (MVIM) is developed to solve fractional integrodifferential equations with nonlocal boundary conditions. It is shown that by choosing suitable initial approximation, the exact solution obtains by one iteration. It is illustrated that the propose method is effective and has high convergency rate.
\end{abstract}

Keywords: Fractional integro-differential equations, nonlocal boundary condition, modification of He's variational iteration method.

\section{Introduction}

The variational iteration method was first proposed by He $[7,8,9]$ and has been worked out over a number of years by many authors. This method has been shown to effectively, easily and accurately solve a large class of nonlinear problems. Generally, one or two iterations lead to high accurate solutions. This method is, in fact, a modification of the general Lagrange multiplier method into an iteration method, which is called correction functional. Applications of the method have been enlarged due to its flexibility, convenience and efficiency. The convergence of the method is systematically discussed by Tatari and Dehghan [17], Odibat [14]. There are several modifications of He's VIM $[6,7,8,9,10]$. In this paper we propose the reliable modification of He's VIM (MVIM) that was introdused by Ghorbani et al. [4] for solving the fractional order integro-differential equations with nonlocal boundary conditions by constructing an initial trial-function without unknown parameters, so that one iteration leads to exact solution.

Consider fractional order integro-differential equation of the form

$$
D^{\alpha} u(x)=g(x)+\int_{a}^{x} h_{1}(x, t) u(t) d t+\int_{a}^{b} h_{2}(x, t) u(t) d t,
$$

where $n-1<\alpha \leq n, \quad a<x<b$, and $n \in N$, with the nonlocal boundary conditions

$$
\sum_{j=1}^{n}\left(\gamma_{i j} u^{(j-1)}(a)+\mu_{i j} u^{(j-1)}(a)\right)+\lambda_{i} \int_{a}^{x} H_{i}(t) u(t) d t=d_{i} \quad i=1 \ldots n
$$

where $D^{\alpha}$ denotes a differential operator with fractional order $\alpha, f(x)$ and $h_{i}(x, t)(i=1,2)$ are holomorphic functions, $H_{i}(t)$ are continuous functions, $\gamma_{i j}, \mu_{i j}, \lambda_{i}$, and $d_{i}(i=1,2, \ldots, m)$ are constants and $u(x)$ is a function of class $C$ (a class of functions that are piecewise continuous on $J^{\prime}:=(0,1)$ and integrable on any finite subinterval $J:=[0,1))$.

It turns out that many problems can be modeled by the fractional order integro-differential equations from various sciences and engineering applications. Furthermore, most problems cannot be solved analytically, hence finding good approximate solutions, using numerical methods, will be very helpful. There are many numerical methods 
for solving these problems, such as Adomian decomposition method [12], fractional differential transform method $[13,1]$, and collocation method [16]. We apply the MVIM for solving these problems. This method solves problems exactly while constructing semi analytic solutions in the polynomial forms.

\section{Basic definitions}

We give some basic definitions and properties of the fractional calculus theory which are used further in this paper.

Definition 2.1: A real function $f(x), x>0$, is said to be in the space $C_{\mu}, \mu \in R$, if there exists a real number $p>\mu$ such that $f(x)=x^{p} f_{1}(x)$, where $f_{1}(x) \in C[0,1)$. Clearly $C_{\mu} \subset C_{\beta}$ if $\beta \leq \mu$.

Definition 2.2: A function $f(x), x>0$, is said to be in the space $C_{\mu}^{m}, m \in N \cup\{0\}$, if $f^{(m)} \in C_{\mu}$.

Definition 2.3: The left sided Riemann -Liouville fractional integral operator of order $\alpha \geq 0$ of a function $f \in C_{\mu}, \quad \mu \geq-1$, is defined as [5]

$$
\begin{aligned}
& J^{\alpha} f(x)=\frac{1}{\Gamma(\alpha)} \int_{0}^{x} \frac{f(t)}{(x-t)^{1-\alpha}} d t, \quad \alpha>0, \quad x>0, \\
& J^{0} f(x)=f(x) .
\end{aligned}
$$

Definition 2.4: Let $f \in C_{-1}^{m}, \quad m \in N \cup\{0\}$, then the Caputo fractional derivative of $f(x)$ is defined as [3,9, 11]

$$
D^{\alpha} f(x)=\left\{\begin{array}{lc}
J^{m-\alpha} f^{(m)}(x), & m-1<\alpha<m, m \in N \\
\frac{D^{m} f(x)}{D x^{m}}, & \alpha=m .
\end{array}\right.
$$

Hence, we have the following properties $[2,3,5,10,11,15,18]$ :

$$
\begin{aligned}
& \text { 1. } J^{\alpha} J^{\nu} f=J^{\alpha+\nu} f, \quad \alpha, \nu>0, f \in C \mu, \mu>0 . \\
& \text { 2. } J^{\alpha} x^{\gamma}=\frac{\Gamma(\gamma+1)}{\Gamma(\alpha+\gamma+1)} x^{\alpha+\gamma}, \quad \alpha>0, \gamma>-1, x>0 . \\
& \text { 3. } J^{\alpha} D^{\alpha} f(x)=f(x)-\sum_{k=0}^{m-1} f^{(k)}\left(0^{+}\right) \frac{x^{k}}{k !}, \quad x>0, \quad m-1<\alpha \leq m .
\end{aligned}
$$

The Caputo fractional derivative [5] is considered here, because it allows traditional initial and boundary conditions to be included in the formulation of the problem. For more information on the mathematical properties of fractional derivatives and integrals, one can consult the mentioned references.

\section{Analysis of the modified variational method}

In this section, for the convenience of the reader, we first present a brief review of He's variational iteration method [6]. Then we will propose the reliable modification of the VIM [4] for solving fractional integro-differential equations with the nonlocal boundary conditions by constructing an initial trial-function without unknown parameters. Here, we consider the following fractional functional equation

$$
L u+R u+N u=g(x),
$$

where $L$ is the fractional order derivative , $R$ is a linear differential operator, $N$ represents the nonlinear terms, and $g$ is the source term. By using (5) and applying the inverse operator $L_{x}^{-1}$ to both sides of (6), and using the given conditions, we obtain

$$
u=f-L_{x}^{-1}[R u]-L_{x}^{-1}[N u]
$$

where the function $f$ represents the terms arising from integrating the source term $g$ and from using the given conditions, all are assumed to be prescribed.

The basic character of He's method is the construction of a correction functional for (6), which reads

$$
u_{n+1}(x)=u_{n}(x)+\int_{0}^{x} \lambda(s)\left[L u_{n}(s)+R \tilde{u}_{n}(s)+N \tilde{u}_{n}(s)-g(s)\right] d s,
$$


where $\lambda$ is a Lagrange multiplier which can be identified optimally via variational theory [6], $u_{n}$ is the $n$th approximate solution, and $\tilde{u}_{n}$ denotes a restricted variation, i.e., $\delta \tilde{u}_{n}=0$.

To solve (6) by He's VIM, we first determine the Lagrange multiplier $\lambda$ that will be identified optimally via integration by parts. Then the successive approximations $u_{n}(x), n \geq 0$, of the solution $u(x)$ will be readily obtained upon using the obtained Lagrange multiplier and by using any selective function $u_{0}$. The approximation $u_{0}$ may be selected by any function that just satisfies at least the initial and boundary conditions. With determined $\lambda$, then several approximations $u_{n}(x), n \geq 0$, follow immediately. Consequently, the exact solution may be obtained by using

$$
\lim _{n \rightarrow \infty} u_{n}(x)=u(x) .
$$

In summary, we have the following variational iteration formula for (7)

$$
\left\{\begin{array}{l}
u_{0}(x) \text { is an arbitrary initial guess, } \\
u_{n+1}(x)=u_{n}(x)+\int_{0}^{x} \lambda(s)\left[L u_{n}(s)+R u_{n}(s)+N u_{n}(s)-g(s)\right] d s,
\end{array}\right.
$$

or equivalently, for (7), according to [19]:

$$
\left\{\begin{array}{l}
u_{0}(x) \text { is an arbitrary initial guess, } \\
u_{n+1}(x)=f(x)-L_{x}^{-1}\left[R u_{n}(x)\right]-L_{x}^{-1}\left[N u_{n}(x)\right]
\end{array}\right.
$$

where the multiplier Lagrange $\lambda$, has been identified.

It is important to note that He's VIM suggests that the $u_{0}$ usually defined by a suitable trial-function with some unknown parameters or any other function that satisfies at least the initial and boundary conditions. This assumption made by He [4, 9] and others will be slightly varied, as will be seen in the discussion.

The MVIM, that was introduced by Ghorbani et al [4], can be established based on the assumption that the function $f(x)$ of the iterative relation (11) can be divided into two parts, namely $f_{0}(x)$ and $f_{1}(x)$. Under this assumption, we set

$$
f(x)=f_{0}(x)+f_{1}(x) .
$$

According to the assumption, (12), and by the relationship (11), we construct the following variational iteration formula

$$
\left\{\begin{array}{l}
u_{0}(x)=f_{0}(x) \\
u_{1}(x)=f(x)-L_{x}^{-1}\left[R f_{0}(x)\right]-L_{x}^{-1}\left[N f_{0}(x)\right] \\
u_{n+1}(x)=f(x)-L_{x}^{-1}\left[R u_{n}(x)\right]-L_{x}^{-1}\left[N u_{n}(x)\right]
\end{array}\right.
$$

where the multiplier Lagrange, $\lambda$, has been identified. Here, a proper selection was proposed for the components $u_{0}(x)$ and $u_{1}(x)$. The suggestion was that only the part $f_{0}$ be assigned to the zeroth component i.e $u_{0}$. An important observation that can be made here is that the success of the proposed method depends mainly on the proper choice of the functions $f_{0}$ and $f_{1}$. As will be seen from the examples below, this selection of $u_{0}$ will result in a reduction of the computational work and accelerate the convergence. Furthermore, this proper selection of the components $u_{0}$ and $u_{1}$ may provide the solution by using one iteration only. To give a clear overview of the content of this study, we have chosen several fractional integro-differential equations with the nonlocal boundary conditions.

\section{Some examples}

In this section, to demonstrate the efficiency of this modification, we solve some examples that are almost no solvable or low accuracy solvable with the original He's VIM. For these propose, we choose several the fractional order integro-differential with nonlocal boundary conditions.

Example 4.1 Consider the following linear fractional integro-differential equation with the given nonlocal conditions [13]

$$
\begin{aligned}
& D^{\frac{1}{2}} y(x)=-x^{2} \frac{e^{x}}{3} y(x)+\frac{1}{\Gamma\left(\frac{3}{2}\right)} x^{\frac{1}{2}}-\frac{1}{2} x^{2}+e^{x} \int_{0}^{x} t y(t) d t+\int_{0}^{1} x^{2} y(t) d t, \\
& y(0)+y(1)-3 \int_{0}^{1} t y(t) d t=0 .
\end{aligned}
$$


By assuming $L=D^{\frac{1}{2}}$ and applying the inverse operator $L_{x}^{-1}$ to both sides of (14), we have

$$
y(x)=J^{\frac{1}{2}}\left[\frac{1}{\Gamma\left(\frac{3}{2}\right)} x^{\frac{1}{2}}-\frac{1}{2} x^{2}\right]+J^{\frac{1}{2}}\left[-x^{2} \frac{e^{x}}{3} y(x)\right]+J^{\frac{1}{2}}\left[e^{x} \int_{0}^{x} t y(t) d t+\int_{0}^{1} x^{2} y(t) d t\right] .
$$

According to the original VIM (10), and corresponding the recursive scheme (11) and by using (5), we obtain

$$
f(x)=f_{0}(x)+f_{1}(x)=J^{\frac{1}{2}}\left[\frac{1}{\Gamma\left(\frac{3}{2}\right)} x^{\frac{1}{2}}-\frac{1}{2} x^{2}\right]=x-\frac{x^{\frac{5}{2}}}{\Gamma\left(\frac{7}{2}\right)},
$$

by assuming

$$
f_{0}(x)=x, \quad f_{1}(x)=-\frac{x^{\frac{5}{2}}}{\Gamma\left(\frac{7}{2}\right)},
$$

and with starting of the initial approximation $y_{0}(x)=f_{0}(x)=x$, we have

$$
\begin{aligned}
y_{0}(x) & =x, \\
y_{1}(x) & =x-\frac{x^{\frac{5}{2}}}{\Gamma\left(\frac{7}{2}\right)}+L_{x}^{-1}\left[f_{0}(x)\right], \\
& =x-\frac{x^{\frac{5}{2}}}{\Gamma\left(\frac{7}{2}\right)}+J^{\frac{1}{2}}\left[\frac{-x^{3}}{3} e^{x}\right]+J^{\frac{1}{2}}\left[\frac{x^{3}}{3} e^{x}\right]+J^{\frac{1}{2}}\left[\frac{x^{2}}{2}\right]=x, \\
y_{n+1}(x) & =x-\frac{x^{\frac{5}{2}}}{\Gamma\left(\frac{7}{2}\right)}+L_{x}^{-1}\left[y_{n}(x)\right] \Rightarrow y_{n+1}(x)=x, \quad n \geq 1 .
\end{aligned}
$$

In view of (18), it is obtained

$$
y(x)=x,
$$

where is the exact solution of (14).

This example supports our aforementioned conclusion that the power and effective of the modified method depends mainly on the proper selection of $f_{0}$ and $f_{1}$. In addition, this confirms our belief that the modified technique accelerates the convergence of the solution by employing only one iteration.

Example 4.2 Consider the following fractional integro-differential equation [13]

$$
D^{\frac{5}{4}} y(x)=(\cos (x)-\sin (x)) y(x)+g(x)+\int_{0}^{x} \sin (t) y(t) d t,
$$

with the nonlocal conditions

$$
\begin{aligned}
& y(0)+y(1)+\frac{e+1}{e+2} y^{\prime}(0)+\frac{1}{2} y^{\prime}(1)-8 \int_{0}^{1} t y(t) d t=0, \\
& 2 y(0)+2 y(1)+\frac{e}{e+1} y^{\prime}(0)-y^{\prime}(1)=0 .
\end{aligned}
$$

By choosing $f(x)=\frac{8}{3} \frac{x^{\frac{3}{4}}}{\Gamma\left(\frac{3}{4}\right)}-2 \cos (x)-2 x \sin (x)+x^{2} \sin (x)+2$, the exact solution of problem (20)-(21) would be $y(x)=x^{2}$.

By using (5) we have

$$
\begin{aligned}
f(x)= & L_{x}^{-1}[g(x)]=J^{\frac{5}{4}} g(x)=\frac{8}{3} \frac{1}{\Gamma\left(\frac{3}{4}\right)} \frac{\Gamma\left(\frac{7}{4}\right)}{\Gamma(3)} x^{2} \\
& -2 J^{\frac{5}{4}} \cos (x)-2 J^{\frac{5}{4}} x \sin (x)+J^{\frac{5}{4}} x^{2} \sin (x)+J^{\frac{5}{4}} 2 \\
= & x^{2}-2 J^{\frac{5}{4}} \cos (x)-2 J^{\frac{5}{4}} x \sin (x)+J^{\frac{5}{4}} x^{2} \sin (x)+J^{\frac{5}{4}} 2 .
\end{aligned}
$$

By selecting $f_{0}(x)=x^{2}$, where is satisfies in nonlocal boundary conditions also, we have

$$
\begin{aligned}
& f_{0}(x)=x^{2} \\
& f_{1}(x)=2 J^{\frac{5}{4}} \cos (x)-2 J^{\frac{5}{4}} x \sin (x)+J^{\frac{5}{4}} x^{2} \sin (x)+J^{\frac{5}{4}} 2 .
\end{aligned}
$$


Following the recursive relationship (13), we obtain

$$
\left\{\begin{array}{l}
y_{0}(x)=f_{0}(x) \\
y_{1}(x)=f(x)-L_{x}^{-1}\left[(\cos (x)-\sin ) x^{2}+\int_{0}^{x} \sin (t) t^{2} d t\right] \\
y_{n+1}(x)=f(x)-L_{x}^{-1}\left[(\cos (x)-\sin ) y_{n}(x)+\int_{0}^{x} \sin (t) y_{n}(t) d t\right], \quad n \geq 1
\end{array}\right.
$$

Hence

$$
\begin{aligned}
& y_{0}(x)=x^{2} \\
& y_{1}(x)=x^{2} \\
& y_{n+1}(x)=x^{2}, \quad n \geq 1 .
\end{aligned}
$$

So

$$
y(x)=\lim _{n \rightarrow \infty} y_{n}(x)=x,
$$

where is the exact solution of problem (20)-(21).

Example 4.3 Consider the following linear fractional integro-differential equation with the given nonlocal condition $[13]$

$$
\begin{aligned}
& D^{\frac{1}{3}} y(x)=\frac{3}{2} \frac{x^{\frac{2}{3}}}{\Gamma\left(\frac{2}{3}\right)}-1+e^{x^{2}}-x^{2} e^{x^{2}}+\int_{0}^{x} x^{2} e^{x t} y(t) d t \\
& y(0)+2 y(1)+3 \int_{0}^{1} t y(t) d t=3 .
\end{aligned}
$$

By using the modified technique, assuming $f_{0}(x)=x$ and $f_{1}(x)=-J^{\frac{1}{3}} 1-J^{\frac{1}{3}} e^{x^{2}}--J^{\frac{1}{3}} x^{2} e^{x^{2}}$, and choosing $y_{0}(x)=f_{0}(x)=x$, we have

$$
\begin{aligned}
& y_{0}(x)=x, \\
& y_{1}(x)=x-J^{\frac{1}{3}} 1-J^{\frac{1}{3}} e^{x^{2}}-J^{\frac{1}{3}} x^{2} e^{x^{2}}-L_{x}^{-1}\left[\int_{0}^{x} x^{2} e^{x t} t d t\right]=x, \\
& y_{n+1}(x)=x, \quad n \geq 1 .
\end{aligned}
$$

In view of (26), it follows that

$$
y(x)=x,
$$

where is the exact solution of problem (25).

Example 4.4 Consider the following linear fractional integro-differential equation with the boundary condition [16]

$$
\begin{aligned}
& D^{\frac{3}{4}} y(x)=\frac{-x^{2} e^{x}}{5} y(x)+\frac{6 x^{\frac{9}{4}}}{\Gamma\left(\frac{13}{4}\right)}+\int_{0}^{x} t e^{x} y(t) d t \\
& y(0)+y(1)-\frac{e+1}{e-2} y^{\prime}(0)+\frac{1}{6} y^{\prime \prime}(1)-5 \int_{0}^{1} t y(t)=0 .
\end{aligned}
$$

By letting, $f_{0}(x)=x^{3}$ and $f_{1}(x)=0$, we select $y_{0}(x)=f_{0}(x)=x^{3}$ to find

$$
\begin{aligned}
& y_{0}(x)=x^{3}, \\
& y_{1}(x)=x^{3}+J^{\frac{3}{4}} \frac{-x^{2} e^{x}}{5} x^{3}+J^{\frac{3}{4}} \frac{6 x^{\frac{9}{4}}}{\Gamma\left(\frac{13}{4}\right)}+J^{\frac{3}{4}} \int_{0}^{x} t e^{x} t^{3} d t=x^{3}, \\
& y_{n+1}(x)=x^{3} . \quad n \geq 1 .
\end{aligned}
$$

In view of $(28)$, it follows that

$$
y(x)=x^{3},
$$

where is the exact solution of problem (27).

It is noteworthy to mentioned here that the exact solution was determined by using only one iteration. 


\section{Conclusion}

In this work, we proposed the reliable modification of He's variational iteration method for solving fractional integrodifferential equations with nonlocal boundary conditions. It is illustrated that the method is power and effective. Furthermore, it accelerates the rate of convergence.

\section{References}

[1] A. Arikoglu, I. Ozkol, Solution of fractional integro-differential equations by using fractional differential transform method, Chaos, Solitons and Fractals (2007).

[2] A. Carpinteri, F. Mainardi, Fractals and Fractional Calculus in Continuum Mechanics, Springer Verlag, Wien, New York, 1997.

[3] K. Diethelm, An algorithm for the numerical solution of differential equations of fractional order, Electron. Trans. Numer. Anal. 5 (1997) 1-6.

[4] A. Ghorbani, J. Saberi-Nadjafi, An effective modification of He's variational iteration method, Nonlinear Analysis: Real World Applications 10 (2009) 2828-2833.

[5] R. Gorenflo, F. Mainardi, Fractional calculus: integral and differential equations of fractional order, in: A. Carpinteri, F. Mainardi (Eds.), Fractals and Fractional Calculus in Continuum Mechanics, Springer, New York, 1997, pp. 223-276.

[6] J. H. He, Some applications of nonlinear fractional differential equation and their approximations, Bull. Sci. Technol. 15 (12) (1999) 86-90.

[7] J. H. He, X .H. Wu, Variational iteration method for autonomous ordinary differential systems, Appl. Math. Comput. 114 (2000) 115-123.

[8] J. H. He ,Variational iteration method for delay differential equations, Commun. Nonlinear Sci. Numer. Simul. 2 (4) (1997) 235-236.

[9] J. H. He, Variational iteration method: New development and applications, Comput. Math. Appl. doi:10.1016/j.camwa. 2006.12 .083 .

[10] J. H. He, Variational iteration method-Some recent results and new interpretations, J. Comput. Appl. Math, 207 (2007) $3-17$.

[11] F. Mainardi, Fractional calculus: some basic problems in continuum and statistical mechanics, in: A. Carpinteri, F. Mainardi (Eds.), Fractals and Fractional Calculus in Continuum Mechanics, Springer, New York, 1997, pp. $291-348$.

[12] S. Momani, M. A. Noor, Numerical methods for fourth order fractional integro-differential equations, Appl. Math. Comput, 182 (2006) 754-60.

[13] D. Nazari , S. Shahmorad, Application of fractional differential transform method to the fractional order integrodifferential equations with nonlocal boundary condition, J. Comput. Appl. Math, (2010), doi:10.1016/j.cam.2010.01.053.

[14] Z. M. Odibat, A study on the convergence of variational iteration method, Mathematical and Computer Modelling (2010), doi:10.1016/j.mcm.2009.12.034.

[15] K .B. Oldham, J. Spanier, The Fractional Calculus, Academic Press, New York, 1974.

[16] E. A. Rawashdeh, Numerical solution of fractional integro-differential equations by collocation method, Appl. Math. Comput., 176 (2006) 1-6.

[17] M. Tatari, M. Dehghan, On the convergence of He's variational iteration method, J. Comput. Appl. Math. 207 (2007) 121-128.

[18] S. Q. Wang , J. H. He, Variational iteration method for solving integro-differential equations, Phys. Lett. A 367 (2007) $188-191$.

[19] L. Xu, Variational iteration method for solving integral equations, Comput. Math. Appl. doi:10.1016/j.camwa.(2006).12.053.

[20] R. Yulita Molliq, M. S. M. Noorani, I. Hashim, Variational iteration method for fractional heat- and wave-like equations, Nonlinear Analysis: Real World Applications 10 (2009) 18541869. 